\title{
Article
}

\section{Respiratory Rate Estimation Based on Spectrum Decomposition}

\author{
SeungJae Lee and Soo-Yong Kim * (i) \\ Samsung Electronics Co., Ltd. \\ * Correspondence: odin.kim@samsung.com; Tel.: +82-31-8037-5438
}

\begin{abstract}
We propose an electrocardiogram (ECG) signal-based algorithm to estimate the respiratory rate is a significant informative indicator of physiological state of a patient. The consecutive ECG signals reflect the information about the respiration because inhalation and exhalation make transthoracic impedance vary. The proposed algorithm extracts the respiration-related signal by finding out the commonality between the frequency and amplitude features in the ECG pulse train. The respiration rate can be calculated from the principle components after the procedure of the singular spectrum analysis. We achieved 1.7569 breaths per min of root-mean-squared error and 1.7517 of standard deviation with a 32-seconds signal window of the Capnobase dataset, which gives notable improvement compared with the conventional Autoregressive model based estimation methods.
\end{abstract}

Keywords: respiratory sinus arrhythmia (RSA), R-peak amplitude (RPA), QRS amplitude

\section{Introduction}

Respiratory rate is one of the significant vital signs to indicate clinical deterioration[1,2]. Even though continuous monitoring of respiration rate is obviously helpful to track the health condition of patients in hospital, home and community settings[3], it is still often not recorded. In [4], this fact is due to the lack of time and clinical knowledge from nurses (usually, the respiratory rate is estimated through stethoscope or by counting the chest wall movements.) as well as the lack of a reliable respiratory rate monitoring devices.

Recent studies have tried to estimate the respiratory rate derived from physical or physiological signal changes due to the respiration activity. There are several ways to estimate the resiratory rate by using physical signal changes. The spirometry[5] can help calculating the respiratory rate as it can measure the flow of air in and out of the mouth during breathing. However, this method need extra equipment to measure air flow in and out. Another sensor for catching the physical signal changes resulted from the respiration, the accelerometer, which can measure acceleration and estimate the quantity of movements, is tried to apply the respiratory rate estimation in non-invasive way[6,7]. The accelerometer is too sensitive to movement noise. For more robust estimation, it is required to apply extra sensors or information.

Another type of solution for detecting the respiration activity is catching the physiological signal changes. The impedance pneumography technique[8] can measure the thoracic volume changes during the respiratory cycle by passing the low-amplitude, high-frequency current between two ECG electrodes. Even though it is currently known as the most reliable technique for measuring the respiratory rate, it has worse signal-to-noise ratio. The ECG signal also has the reflection of the respiration activity. Due to the respiration activity, there are three types of modulation can be observed[9] : baseline wander, amplitude modulation and frequency modulation. By using the phenomena, several studies have shown the ways to extract the respiratory signal from the modulated signals through autoregressive modeling methods[4,10-12]. In [13], it introduces that variations of the angle of mean electrical axis (AMEA) of the heart reflect the respiratory patterns. 
In this paper, we focus on the respiratory rate estimation algorithm based on single-lead ECG signals. There are several benefits to utilize single-lead ECG. First, a single-lead ECG system can be easily incorporated in a wearable type device. A single-lead ECG system gives higher comfortability compared with other complicated sensors include multi-lead ECG system. Second, the device has an ability to monitor cardiac and respiratory activities simultaneously.

The remaining part of paper is organized as follows. Section2 describes physiological relationship between ECG signal and respiration. We also introduce some kinds of approaches about extracting the respiration signal from the given ECG signal. Section 3 depicts our proposed algorithm for estimating respiratory rate in a fusion way. Section 4 shows the results of the performance analysis of our proposed algorithm. Section 5 gives a discussion and a conclusion of this paper.

\section{Preliminaries}

Respiration affects ECG signals in several ways : baseline wander, amplitude modulation and frequency modulation[9]. Generally, the frequency range of respiration is located between of 0.1 and $0.5 \mathrm{~Hz}[14]$. The frequency of the respiration activity on the baseline of the ECG signals is easily contaminated by various types of artifacts such as movement artifacts, which is not predicted. Due to the characteristics, the baseline wandering effect is not suitable for utilizing to estimate respiratory rate, hence, many studies used to concentrate on the modulated signals to obtain respiratory rate from ECG signals.

Respiration is known to modulate the heart rate such that it increases during inspiration and decreases during expiration[14]. It is similar principle with frequency modulation, thus a waveform can be extracted from the heart rate time series representing this modulation, which is referred to as respiratory sinus arrhythmia (RSA). Many studies have focused on estimating a dominant frequency in the modulated signals as an index of respiratory activity[11,15,16].

There is another signal, which is reflected on the respiration. During inspiration and expiration, the filling and emptying of the lungs causes a rotation of the electrical axis of the heart and a change in the impedance of the thorax. It yields changes in the electrocardiogram beat morphology. Consequently, the R-peak amplitudes are modulated by the respiratory activity in an amplitude modulation manner[14]. Respiratory rate can be derived from this modulation, which is referred as the R-peak amplitude (RPA).

In order to estimate respiratory rate from the extracted RPA and RSA, spectral information related to the respiration should be used. The main assumption is that the dominant frequency of the given signals is regards as the respiration related signal. Common approach to obtain the respiration-related frequency in RPA and RSA is spectral analysis or time-spectral analysis.

The traditional technique to find a dominant spectral peak in a given signal is a Fourier Transform-based method[17]. It is simple, but it should be assumed that the signal is stationary or locally stationary. Further, it can't extract the cluttered spectral information. In contrast, Autoregressive (AR) spectral modeling[18-20] gives clean and uncluttered spectral information. However, the result of AR modeling reflects not only the input, but also assumptions about its complexity.

Another approach is a time-frequency analysis such as continuous wavelet transform[21] or empirical mode detection[17,22]. It tries to decompose the signal first, then, find a dominant spectral peak in a decomposed signal.

Both of the traditional spectral analysis and the AR modeling method requires uniform sampling. However, the RSA and the RPA signals are non-uniform sampled signals. Hence, interpolation is required to analyze the spectral information of the signals. Linear interpolation or cubic spline interpolation technique is traditional way of interpolation. The study by Berger[23] provides a good performance in the ECG analysis.

Since the respiration-related activity is generally located at the range of 0.1 and $0.5 \mathrm{~Hz}$, the RPA and RSA signals are downsampled at 2 or $4 \mathrm{~Hz}$. Especially, this downsampling gives an improvement of the resolution of the phase angles and identifying the one corresponding to the respiratory frequency[4]. 


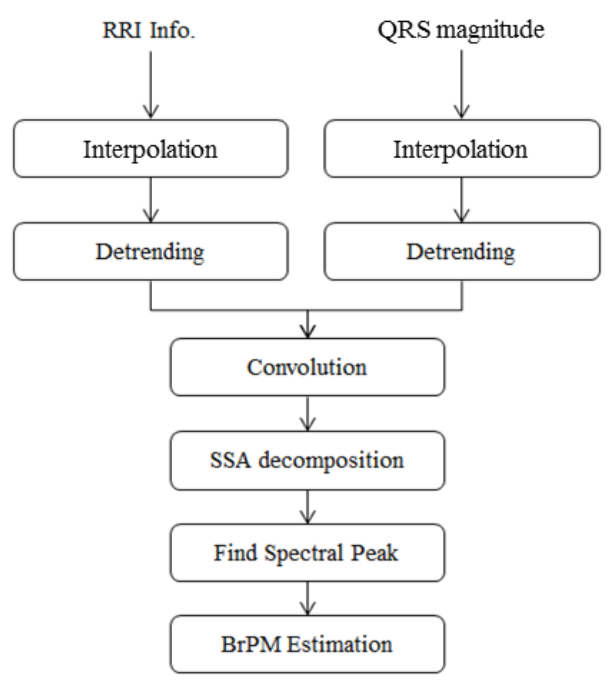

Figure 1. The procedure of the proposed algorithm

\section{Spectrum Decomposition Algorithm for Respiratory Rate}

In this paper, our proposed algorithm named as spectrum decomposition algorithm for respiratory rate (SDAR), focuses on the respiratory rate estimation based on spectrum decomposition with a single-lead ECG signal. As described in Section 2, respiratory rate can be derived from the relationship between respiration and the modulated signals. In the SDAR algorithm, a FM modulated signal, RSA and an AM modulated signal, QRS amplitude is utilized to estimate respiratory rate. Many studies used to utilize RPA, however, it is sensitive to noise and motion artifacts. To reduce the effects of the artifacts, the SDAR algorithm uses the difference of the maximum and the minimum of QRS complexes, which is defined as QRS amplitude, instead of using the RPA directly. For obtaining an accurate estimation result, the SDAR algorithm is based on fusion techniques. The SDAR algorithm employs the convolutional method to extract the common frequency component between the RSA signal and the QRS amplitude based signal.

\subsection{Extracting Modulated Signals}

The SDAR algorithm utilizes QRS amplitude and RSA from a given ECG signal. At the begin of the algorithm, QRS complexes should be detected. The SDAR algorithm employs Pan and Tompkins QRS complex detector[24], which is one of the widely used as the peak detector. After obtaining the R peak indices, RSA signal can be easily organized by R to R inter-beat intervals with the corresponding time indices. QRS amplitude is calculated by Eq.1.

$$
Q R S_{a m p}^{i}=\max _{t \in S} E C G_{S}-\min _{t \in S} E C G_{S}
$$

where $i$ is the index of the given peak data, $S$ is a set of data in the searching window, which has width $w$ centered at the $i$ th peak, $t$ means the timing index within the searching window $S$ and $E C G_{S}$ depicts ECG signals within searching window $S$.

The SDAR algorithm is based on a fusion technique with both of modulated signals. To obtain the spectral information from the modulated signals, it should make the signals uniform sampled. The algorithm applies interpolation to each of RSA and QRS amplitude. Berger's method[23] is applied for interpolating RSA signal and spline interpolation is applied for obtaining uniformly sampled QRS amplitude signal. The results of the interpolation from a given ECG signal are shown in Fig.2. 


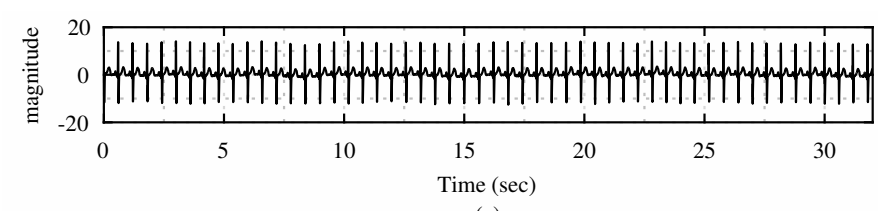

(a)

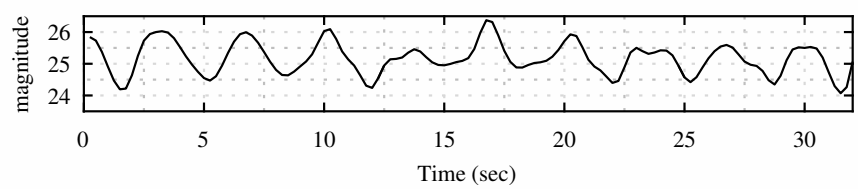

(b)

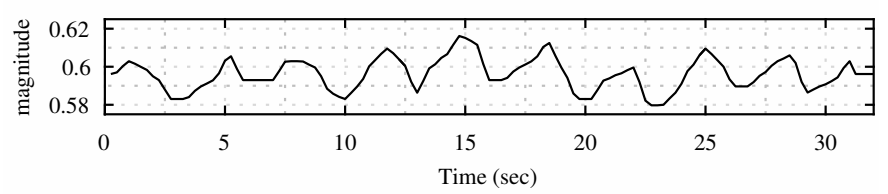

(c)

Figure 2. QRS amplitude and RSA signals extracted from a given ECG signal (a) ECG signal (b) interpolated QRS amplitude (c) RSA

\subsection{Detrending}

Spectral analysis inherently assumes that the signal is weakly stationary at least[25]. However, heart rate variability (HRV) signal such as RSA and QRS amplitude is not stationary[26]. Detrending is the process to remove the non-stationarities in the signal. In this paper, The SDAR algorithm employs a detrending method using smoothness prior approach (SPA) [25]. According to the SPA, a series of data can be written into stationary part and non-stationary part as

$$
z=z_{\text {stat }}+z_{\text {trend }}
$$

where $z_{\text {stat }}$ implies the stationary part and $z_{\text {trend }}$ is the non-stationary part of the given series of data. The non-stationary component $z_{\text {trend }}$ can be modeled with a linear observation model as

$$
z_{\text {trend }}=H \theta+v
$$

where $H \in \mathbb{R}$ is the observation matrix, $\theta \in \mathbb{R}$ are the regression parameters and $v$ is the observation error. The estimation of the regression parameter $\hat{\theta}$ can be obtained by the regularized least squares method.

$$
\hat{\theta}=\arg \min _{\theta}\left\{\|H \theta-z\|^{2}+\lambda^{2}\left\|D_{d}(H \theta)\right\|^{2}\right\}
$$

As shown in Fig.3, the extracted signals have trend components. The dashed line of Fig.3 (a) and (c) is the trending line by the SPA method. After subtracting the fitting line from the extracted signals, the detrended signals are shown in Fig.3 (b) and (d).

\subsection{Normalization and Convolution}

The SDAR algorithm is based on the fusion technique using both of RSA and QRS amplitude information. Since the energy level of the extracted signals is different each other, normalization is required to avoid a biased result. In order to make the signals same energy levels, each signal is divided by the root mean square (RMS) of the signal.

After the normalization, our algorithm takes the convolution of detrended QRS amplitude and detrended RSA signals. The convolution makes the common frequency components salient and suppresses the frequency components which are not observed in common in the RSA and QRS amplitude signals. To reduce the edge effect of the signal, the algorithm takes a hanning window 

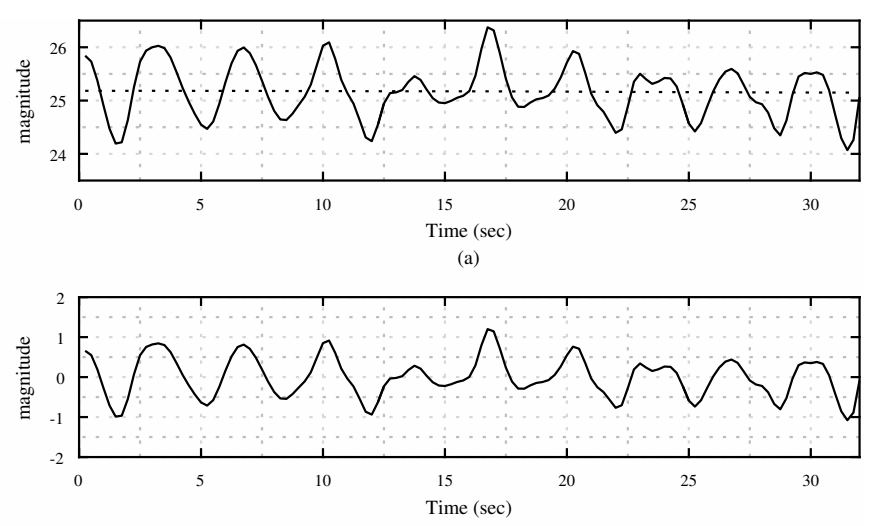

(b)

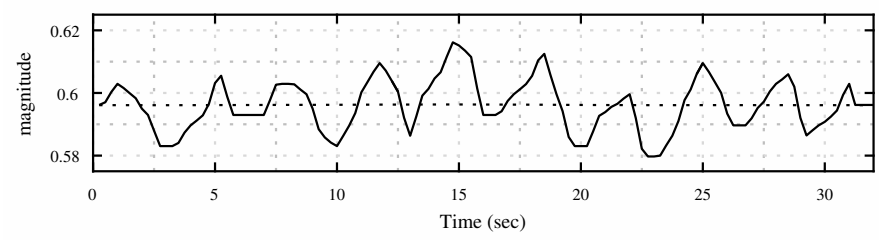

(c)

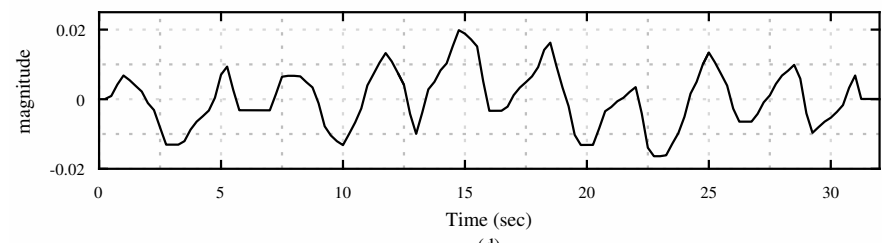

Figure 3. Detrending (a) QRS amplitude without detrending and fitting line (dashed) (b) detrended QRS amplitude (c) RSA without detrending and fitting line(dashed) (d) detrended RSA 

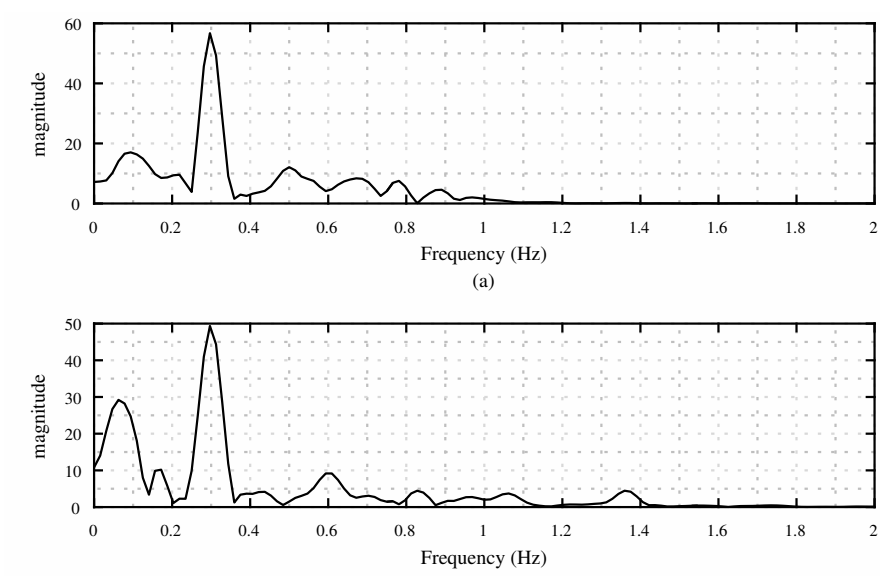

(b)

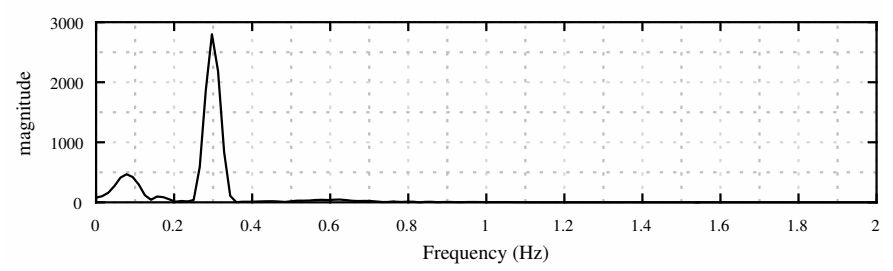

(c)

Figure 4. Fusion (a) spectrum of normalized QRS amplitude (b) spectrum of normalized RSA (c) spectrum of convolution

before convolution. Fig.4 depicts the normalized signals and convolution of QRS amplitude and RSA signals.

\subsection{Singular Spectrum Analysis}

The singular spectrum analysis (SSA) technique is a powerful technique of time series analysis incorporating the elements of classical time series analysis, multivariate statistics, multivariate geometry, dynamical systems and signal processing $[27,28]$. The aim of SSA is to make a decomposition of the original series into the sum of a small number of independent and interpretable components.

The SSA comprises two steps, decomposition and reconstruction steps. At decomposition step, one-dimensional time series $Y_{T}=\left(y_{1}, \ldots, y_{T}\right)$ are transferred into the multi-dimensional series $X_{1}, \ldots, X_{T}$ with vectors $X_{i}=\left(y_{i}, \ldots, y_{i+L-1}\right)^{\prime}$. This process is named as embedding. The single parameter of the embedding is the window length $L(2 \leq L \leq T)$. After embedding, the trajectory matrix $X=\left[X_{1}, \ldots, X_{K}\right]$ is obtained. Decomposition is based on singular value decomposition (SVD) of the obtained trajectory matrix. After SVD, the trajectory matrix is decomposed into rank 1 matrices, and the vectors of principle components $(P C s)$ by the eigentriples, $\left(\sqrt{\lambda_{i}}, U_{i}, V_{i}\right)$.

Fig. 5 shows the principle components, which have the largest eigenvalues in numerical order, decomposed by SSA.

According to the applications, the reconstruction steps is required. However, our main goal to use SSA in our application decomposes the given signal in independent time series.

\subsection{Finding a spectral peak}

The principle components obtained at the SSA decomposition step should be sorted in numerical descending order. That means the first principle component has largest energy occupied in a given signal. Our assumption is that the RSA and QRS amplitude have respiration-related frequency components, which are dominant in the signal. Hence, the dominant frequency of the first principle component could be the respiration-related frequency. 

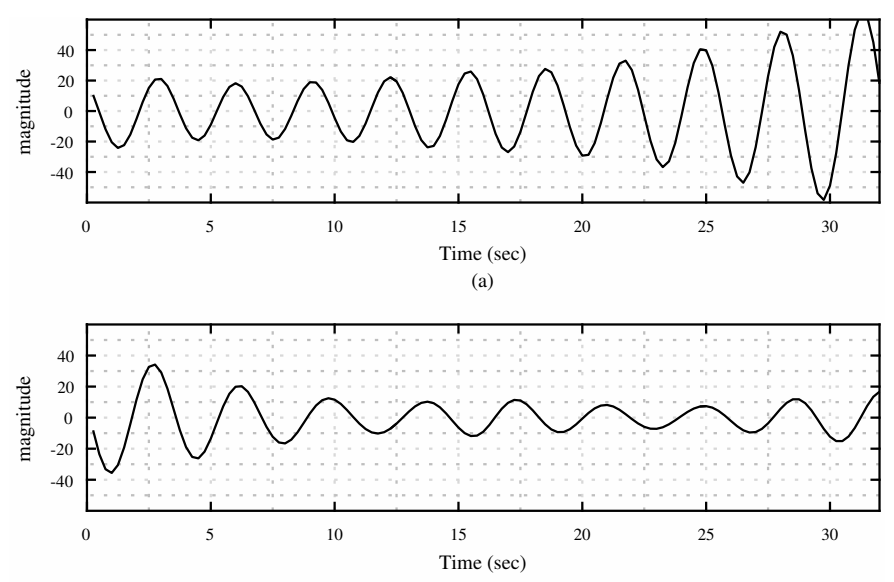

(b)

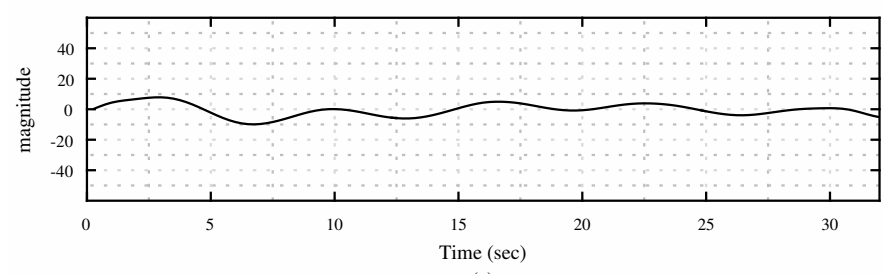

(c)

Figure 5. Decomposed time series signals by SSA

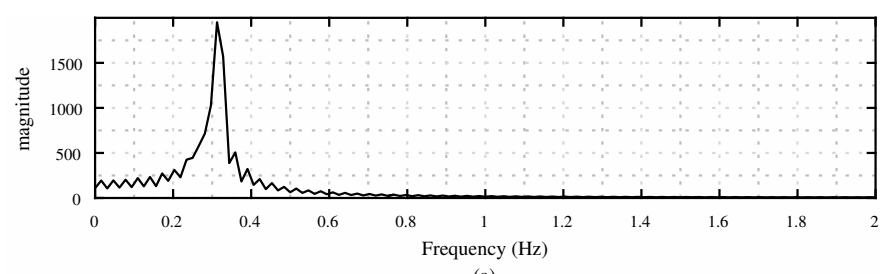

(a)
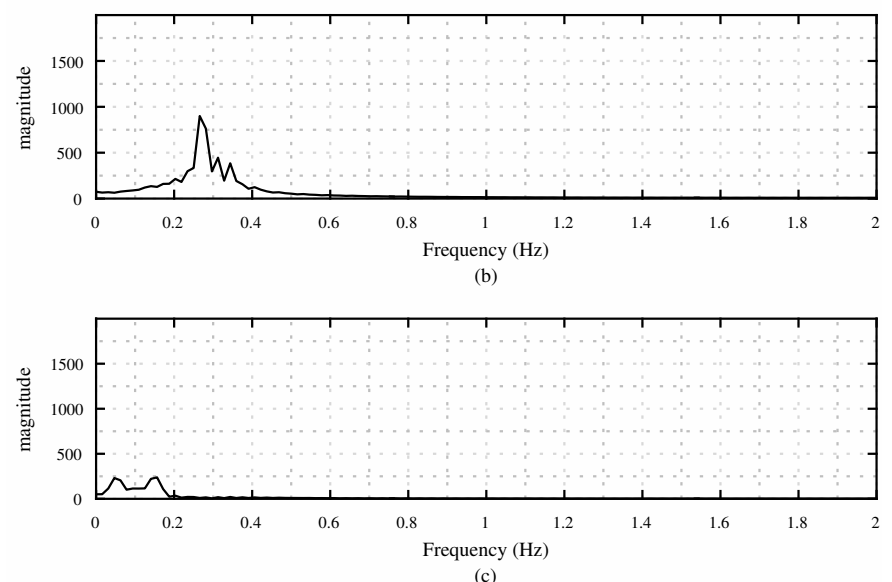

Figure 6. Power spectral density of the SSA decomposed signals 
To search for the spectral peak of respiratory activities, the algorithm should transform the time series principle components into spectral domain. Fig.6 shows the transformed principle components.

The SSA decomposition may make the given signal split into different principle components, even though they have similar range of frequency components. Further, if there are some dominant components our of range of the respiration $(0.1 \sim 0.7 \mathrm{~Hz})$, the first principle component may not be the component which has a respiratory signal. It can result in the error in estimation. In order to avoid estimation error, our algorithm selects five of the most influential principle components and reconstructs the signal to find the spectral peak.

\section{Simulation Results and Analysis}

\subsection{Database used}

In this paper, Capnobase 8-min database, which is available for download from the online database[29], is used to evaluate the algorithm. The database is composed of forty two 8-min segments from 29 pediatrics and 13 adults cases. Each recording contains raw PPG and ECG signals with annotations by an expert rater, reference $\mathrm{CO}_{2}$ signals and derived instantaneous respiration rate for all cases. The capnometric waveform was used as the reference gold standard recording for estimating respiration rate. The evaluation is performed with 35 recordings out of 42 recordings, since they have signal distortion or out of respiration rate range $(0.1 \sim 0.7 \mathrm{~Hz})$.

\subsection{Parameters}

Generally, human respiratory frequency is located at the range of 0.1 and $0.5 \mathrm{~Hz}[14]$. In this paper, the frequency region of interest is set to 0.1 and $0.7 \mathrm{~Hz}$, since the capnometric waveform in Capnobase dataset, is observed at the range of 0.1 and $0.7 \mathrm{~Hz}$. Further, 32-sec window is used as the time window for obtaining the modulated signals. The modulated signals are extracted and interpolated at $4 \mathrm{~Hz}$, hence 128-point interpolated signals are obtained in time-frequency analysis.

\subsection{Evaluation}

The SDAR is compared with the conventional respiratory rate estimation algorithms, which are based on AR methods using RPA and RSA. The comparison is operated with AR with RPA only, AR with RSA only, AR with fusion technique[4] and SDAR (proposed). The respiratory rate estimation result of each method is shown in Fig.7. A solid gray line represents the reference respiratory rate based on the capnometry signal. Dashed lines demonstrate the estimation results by the conventional methods based on AR modeling. Single modulated signal based method such as AR with RPA only or AR with RSA only shows more unstable estimation results than fusion-based method. AR with fusion technique shows better performance than the single modulated signal based method, since it determines the respiratory rate by selecting the better estimation between RPA and RSA. A black solid line, which depicts the estimation results by the SDAR, demonstrate minimum tracking errors out of the given methods as shown in Fig.7.

Over the whole segments in the dataset, the accuracy of the estimation results is shown in Fig.8. Gray markers represent the respiratory rate estimation results based on the conventional methods, and black markers shows the results of SDAR algorithm. In a ideal case, the estimation results should be on the bold gray line in the center of the graph. In other words, the estimation accuracy is superior if the estimation results are concentrated on the gray line.

To quantify the performance, RMS error and standard deviation of the error are used as the metric of the performance. RMS is defined as

$$
R M S=\sqrt{\frac{1}{n} \sum_{i=1}^{n}\left(B r_{r e f}^{i}-B r_{e s t}^{i}\right)}
$$



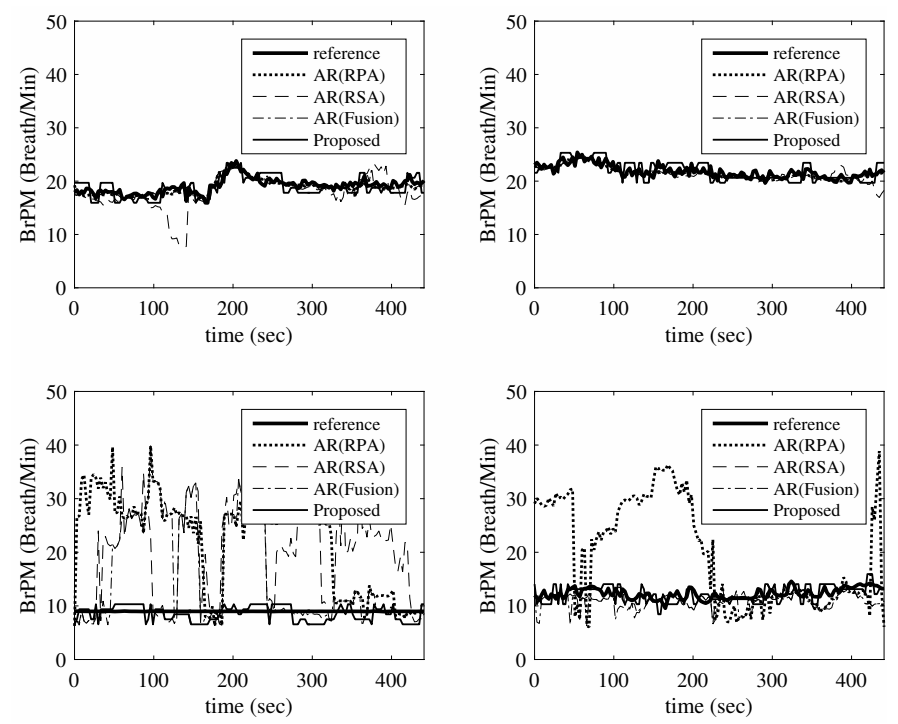

Figure 7. Comparison with conventional respiratory rate estimation algorithm

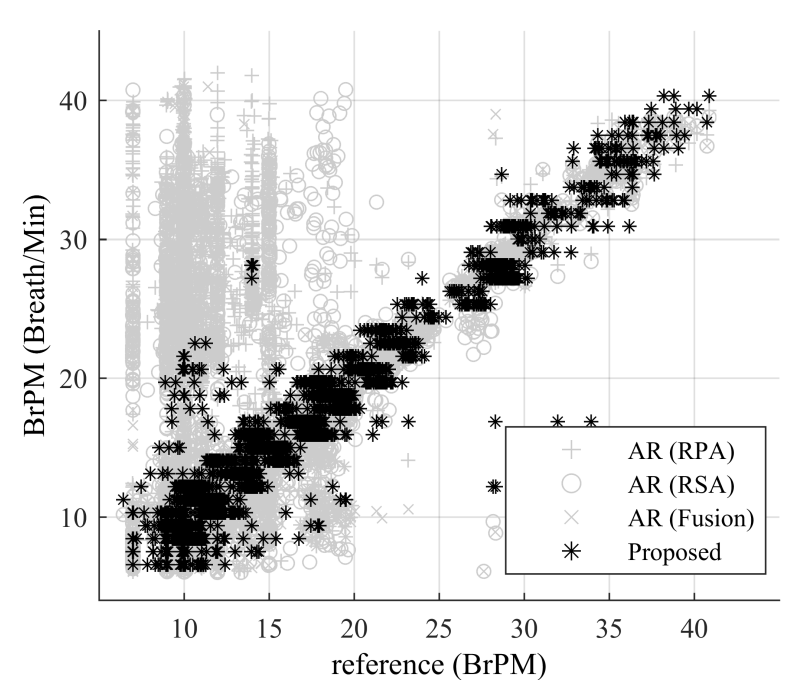

Figure 8. Comparison with conventional respiratory rate estimation algorithm 
Table 1. Performance analysis compared with the conventional methods

\begin{tabular}{l|c|c} 
& RMS Error (Breath/min) & std. (error) \\
\hline & 11.4016 & \\
AR with RPA only & 7.1853 & 9.3401 \\
AR with RSA only & 5.5391 & 6.6895 \\
AR with fusion & 1.7568 & 5.4216 \\
SDAR (proposed) & & 1.7517
\end{tabular}

AMEA Angle of Mean Electrical Axis

AR Autoregressive

ECG Electrocardiogram

HRV Heart Rate Variability

RMS Root Mean Square

232 RPA R-Peak Amplitude

RSA Respiratory Sinus Arrhythmia

SDAR Spectrum Decomposition Algorithm for Respiratory Rate

SPA Smoothness Prior Approach

SSA Singular Spectrum Analysis

SVD Singular Value Decomposition 
233

Braun, S.R. Respiratory rate and pattern 1990.

Ridley, S. The recognition and early management of critical illness. Annals of the Royal College of Surgeons of England 2005, 87, 315 .

3. Williams, B.; Alberti, G.; Ball, C.; Bell, D.; Binks, R.; Durham, L.; others. National Early Warning Score (NEWS): Standardising the assessment of acute-illness severity in the NHS. London: The Royal College of Physicians 2012.

4. $\quad$ Orphanidou, C.; Fleming, S.; Shah, S.A.; Tarassenko, L. Data fusion for estimating respiratory rate from a single-lead ECG. Biomedical Signal Processing and Control 2013, 8, 98-105.

5. Pierce, R.; others. Spirometry: an essential clinical measurement. Australian family physician 2005, $34,535$.

6. Liu, G.Z.; Guo, Y.W.; Zhu, Q.S.; Huang, B.Y.; Wang, L. Estimation of respiration rate from three-dimensional acceleration data based on body sensor network. Telemedicine and e-health 2011, 17, 705-711.

7. Lapi, S.; Lavorini, F.; Borgioli, G.; Calzolai, M.; Masotti, L.; Pistolesi, M.; Fontana, G.A. Respiratory rate assessments using a dual-accelerometer device. Respiratory physiology E neurobiology 2014, 191, 60-66.

8. Larsen, V.H.; Christensen, P.H.; Oxhøj, H.; Brask, T. Impedance pneumography for long-term monitoring of respiration during sleep in adult males. Clinical Physiology 1984, 4, 333-342.

9. Charlton, P.H.; Bonnici, T.; Tarassenko, L.; Clifton, D.A.; Beale, R.; Watkinson, P.J. An assessment of algorithms to estimate respiratory rate from the electrocardiogram and photoplethysmogram. Physiological measurement 2016, 37, 610 .

10. Cysarz, D.; Zerm, R.; Bettermann, H.; Frühwirth, M.; Moser, M.; Kröz, M. Comparison of respiratory rates derived from heart rate variability, ECG amplitude, and nasal/oral airflow. Annals of biomedical engineering 2008, 36, 2085-2094.

11. Schäfer, A.; Kratky, K.W. Estimation of breathing rate from respiratory sinus arrhythmia: comparison of various methods. Annals of Biomedical Engineering 2008, 36, 476-485.

12. Mirmohamadsadeghi, L.; Vesin, J.M. Respiratory rate estimation from the ECG using an instantaneous frequency tracking algorithm. Biomedical Signal Processing and Control 2014, 14, 66-72.

13. Behbehani, K.; Vijendra, S.; Burk, J.; Lucas, E. An investigation of the mean electrical axis angle and respiration during sleep. Engineering in Medicine and Biology, 2002. 24th Annual Conference and the Annual Fall Meeting of the Biomedical Engineering Society EMBS/BMES Conference, 2002. Proceedings of the Second Joint. IEEE, 2002, Vol. 2, pp. 1550-1551.

14. Bailón, R.; Sörnmo, L.; Laguna, P.; others. ECG-derived respiratory frequency estimation. Advanced methods and tools for ECG data analysis Artech House Inc 2006, pp. 215-244.

15. Mason, C.; Tarassenko, L. Quantitative assessment of respiratory derivation algorithms. Engineering in Medicine and Biology Society, 2001. Proceedings of the 23rd Annual International Conference of the IEEE. IEEE, 2001, Vol. 2, pp. 1998-2001.

16. Park, S.B.; Noh, Y.S.; Park, S.J.; Yoon, H.R. An improved algorithm for respiration signal extraction from electrocardiogram measured by conductive textile electrodes using instantaneous frequency estimation. Medical \& biological engineering \& computing 2008, 46, 147-158.

17. Karlen, W.; Raman, S.; Ansermino, J.M.; Dumont, G.A. Multiparameter respiratory rate estimation from the photoplethysmogram. IEEE Transactions on Biomedical Engineering 2013, 60, 1946-1953.

18. Pardey, J.; Roberts, S.; Tarassenko, L. A review of parametric modelling techniques for EEG analysis. Medical engineering $\mathcal{E}$ physics 1996, 18, 2-11.

19. Nemati, S.; Malhotra, A.; Clifford, G.D. Data fusion for improved respiration rate estimation. EURASIP journal on advances in signal processing 2010, 2010, 1.

20. Fleming, S.; Tarassenko, L.; Thompson, M.; Mant, D. Non-invasive measurement of respiratory rate in children using the photoplethysmogram. 2008 30th Annual International Conference of the IEEE Engineering in Medicine and Biology Society. IEEE, 2008, pp. 1886-1889.

21. Addison, P.S.; Watson, J.N. Secondary transform decoupling of shifted nonstationary signal modulation components: application to photoplethysmography. International Journal of Wavelets, Multiresolution and Information Processing 2004, 2, 43-57.

22. Huang, N.E.; Shen, Z.; Long, S.R.; Wu, M.C.; Shih, H.H.; Zheng, Q.; Yen, N.C.; Tung, C.C.; Liu, H.H. The empirical mode decomposition and the Hilbert spectrum for nonlinear and non-stationary time series 
analysis. Proceedings of the Royal Society of London A: Mathematical, Physical and Engineering Sciences. The Royal Society, 1998, Vol. 454, pp. 903-995.

23. Berger, R.D. Analysis of the cardiovascular control system using broad-band stimulation. PhD thesis, Massachusetts Institute of Technology, 1987.

24. Hamilton, P.S.; Tompkins, W.J. Quantitative investigation of QRS detection rules using the MIT/BIH arrhythmia database. IEEE transactions on biomedical engineering 1986, pp. 1157-1165.

25. Tarvainen, M.P.; Ranta-Aho, P.O.; Karjalainen, P.A.; others. An advanced detrending method with application to HRV analysis. IEEE Transactions on Biomedical Engineering 2002, 49, 172-175.

26. Berntson, G.; Bigger Jr, J.; Eckberg, D.; Grossman, P.; Kaufmann, P.; Malik, M.; Nagaraja, H.; Porges, S.; Saul, J.; Stone, P.; others. Heart rate variability: origins, methods, and interpretive caveats. Psychophysiology 1997, 34, 623-648.

27. Broomhead, D.S.; King, G.P. Extracting qualitative dynamics from experimental data. Physica D: Nonlinear Phenomena 1986, 20, 217-236.

28. Hassani, H. Singular Spectrum Analysis: Methodology and Comparison. Journal of Data Science 2007, 5, 239-257.

29. Karlen, W.; Turner, M.; Cooke, E.; Dumont, G.; Ansermino, J. CapnoBase: Signal database and tools to collect, share and annotate respiratory signals. Annual Meeting of the Society for Technology in Anesthesia (STA), West Palm Beach, 2010, p. 25. 